\title{
Una experiencia de formación universitaria en relación con organizaciones sociales. Reflexiones sobre la práctica docente ${ }^{1}$
}

\author{
Ana Luz Abramovich \\ analuzabra@gmail.com
}

Natalia Da Representação

ndarepre@gmail.com

\section{Marisa Fournie}

marisafournier@yahoo.com.ar
Docentes e investigadoras de la Universidad Nacional Genera Sarmiento, Argentina.
Integración de la docencia y la extensión /

Intervenciones

RECEPCIÓN: 24/06/16

ACEPTACIÓN FINAL: 10/10/16

\section{Resumen}

Este artículo presenta reflexiones sobre las implicancias de la tarea docente en una experiencia de vinculación entre la Universidad Nacional de General Sarmiento y organizaciones sociales de la Región Metropolitana de Buenos Aires. El "Laboratorio: Redes Sociales y Condiciones de Vida", que comenzó a funcionar en el año 2005, es una materia obligatoria en la que los estudiantes realizan un trabajo de investigación y/o intervención para las organizaciones sociales. La materia busca que los estudiantes pongan en juego capacidades para el trabajo profesional con grados significativos de reflexión crítica y compromiso social. El rol docente implicado en esta tarea se diferencia del habitual. Supone la capacidad para acompañar el proceso de intercambio y de aprendizaje de los estudiantes y de las organizaciones, la posibilidad de distanciarse de una mirada única para resolver problemas complejos y la capacidad de incorporar la incertidumbre como una variable central del proceso.

Palabras-clave

- Articulación universidad-organizaciones sociales

- Prácticas profesionales

- Tarea docente

- Utilidad social de la investigación

\section{Resumo}

Este artigo apresenta reflexões sobre as implicações da tarefa de ensinar em uma experiência de vinculação entre a Universidade Nacional de General Sarmiento e as organizações sociais da área metropolitana de Buenos Aires. O "Laboratório: Redes sociais e condições de vida", que começou a operar em 2005, é uma disciplina obrigatória no qual os alunos realizam um trabalho de pesquisa e/ou de intervenção para as organizações sociais. A diciplina procura que os estudantes exponham as capacidades para o trabalho profissional com níveis significativos de reflexão crítica e compromisso social. O papel docente envolvido nesta tarefa se diferencia do habitual. Pressupõe a capacidade de acompanhar o processo de intercâmbio e aprendizagem dos estudantes e das organizações, a posibilidade de afastar-se de um olhar único para resolver problemas complexos, e a capacidade de incorporar a incerteza como uma variável central do processo.

Palavras-chave

- Articulação universidade-organizações socias

- Práticas profissionais

- Tarefa docente

- Utilidade social da pesquisa

\section{Para citación de este artículo} Abramovich, A. L., Da Representação, N. y Fournier, M. (2016). Una experiencia de formación universitaria en relación con organizaciones sociales. Reflexiones sobre la práctica docente. En Revista +E versión digital, (6), pp. 404-409. Santa Fe, Argentina: Ediciones UNL. 


\section{Introducción}

Este artículo presenta reflexiones sobre las implicancias de la tarea docente en una experiencia de vinculación entre la Universidad Nacional de General Sarmiento (UNGS) y organizaciones sociales de la Región Metropolitana de Buenos Aires El "Laboratorio: Redes Sociales y Condiciones de Vida", que comenzó a funcionar en el año 2005, es una materia obligatoria en la que los estudiantes realizan un trabajo de investigación y/o intervención para las organizaciones sociales.

En un lapso de cuatro meses y medio se trabaja en la construcción conjunta de un problema identificado por cada organización, que luego es abordado por el grupo de estudiantes, quienes realizan aportes desde el quehacer científico.

Las problemáticas abordadas giran en torno a algunos de estos interrogantes: la forma en que los vecinos del barrio conocen y acceden a los servicios y actividades de la organización, los impactos de su trabajo en las comunidades, la percepción pública sobre las temáticas que abordan, la reconstrucción de su historia, las posibilidades de nuevas vinculaciones y la viabilidad de nuevos proyectos, entre otras. $^{2}$

Los resultados del estudio son devueltos teniendo en consideración los intereses de la organización y las condiciones necesarias para su comunicación en la comunidad de referencia.

El Laboratorio se sitúa en un punto de intersección entre la formación teórica conceptual, la investigación y la acción con la comunidad. La articulación entre estos tres elementos es el eje de estructuración de la propuesta. Un rasgo diferencial está dado por el hecho de que los estudiantes pasan por una experiencia de formación en prácticas profesionales en los inicios de sus trayectos formativos. Este esquema se estructura en lo que se denomina "enseñanza por proyectos".

La materia busca que los estudiantes pongan en juego capacidades para el trabajo profesional con grados significativos de reflexión crítica y compromiso social. El rol docente implicado en esta tarea se diferencia del habitual. Supone la capacidad para acompañar el proceso de intercambio y de aprendizaje de los estudiantes y de las organizaciones, la posibilidad de distanciarse de una mirada única para resolver problemas complejos, y la capacidad de incorporar la incertidumbre como una variable central del proceso.

La interacción entre estudiantes y docentes y el vínculo estrecho que se genera con las organizaciones sociales son parte de ese aprendizaje, que cobra sentido en varios planos: porque se trata de un trabajo real con organizaciones sociales de la región, porque deriva en un producto que tiene utilidad social y porque se configura en relación con las orientaciones y prácticas que marcan el modelo de universidad donde la experiencia tiene lugar. Otro elemento destacable de la propuesta es el trabajo grupal y colectivo que integra distintas miradas, lógicas, formaciones disciplinares e intereses.

\section{El marco institucional en que se desarrolla la propuesta}

La UNGS es una institución pública y estatal fundada en 1993. Está localizada en el noroeste de la Región Metropolitana de Buenos Aires y su área de influencia directa cubre total o parcialmente un amplio espacio que abarca varios municipios del Conurbano Bonaerense: Tigre, Malvinas Argentinas, San Miguel, José C. Paz, Moreno, Hurlingham, Morón, Merlo, Pilar y Escobar. De estos distritos provienen la mayoría de los estudiantes. Desde su creación desarrolló vínculos y articulaciones con diferentes actores que hacen a la vida institucional, económica y política de la región en función del planteamiento de alternativas de desarrollo integral. Tal es así que una parte sustantiva de sus acciones en formación, investigación y servicios está fuertemente ligada a las problemáticas que atraviesan las grandes ciudades en la actualidad, y a las áreas de actuación de las organizaciones sociales. En este sentido, la definición de "qué se enseña y qué se investiga" en función de objetivos estratégicos ha formado y forma parte de las preocupaciones de este espacio académico tanto en el momento de su creación como en la actualidad, con 16 años de funcionamiento.

En este marco, la propuesta pedagógica fundada en el trabajo articulado con las organizaciones sociales responde a los lineamientos constitutivos de la institución y a un área de vacancia en la currícula existente. Se genera, entonces, una propuesta de Laboratorio Interdisciplinario que se cursa en el $2^{\circ}$ año de todas las carreras de la Universidad, lo que surge tanto de una necesidad institucional por cubrir esta temática específica a través de una oferta formativa para todos los estudiantes en temas relativos al desarrollo de las organizaciones sociales en el conurbano bonaerense como por el interés del equipo docente de encauzar en un espacio novedoso los múltiples intercambios realizados en instancias previas con las organizaciones sociales del entorno de la Universidad, las cuales demostraban interés en profundizar alternativas de trabajo conjunto.
1) El artículo sintetiza algunas reflexiones que se encuentran en el libro de Abramovich (2012). Allí puede encontrarse una descripción más detallada de la experiencia junto con el marco teórico de la propuesta pedagógica, así como la evaluación de los estudiantes y las organizaciones que pasaron por la materia hasta el año 2011. Puede descargarse en: http://www.ungs.edu. ar/areas/publicaciones/467/
2) Una descripción exhaustiva de cada uno de los estudios realizados puede leerse en el Capítulo 3 del mencionado libro, donde se recogen testimonios de estudiantes y miembros de las organizaciones. 


\section{6}

el enfoque teórico del Laboratorio

se sostiene en la hipótesis de que es

difícil comprender al mundo asociativo

si no se lo relaciona con los modelos

de desarrollo hegemónicos

\subsection{Presentación de la propuesta pedagógica}

Esta materia busca dar una respuesta a cuestiones claves vinculadas con:

a) La creación de un espacio novedoso de articulación duradera entre la universidad y las organizaciones sociales, a partir de una propuesta pedagógica concreta.

b) La necesidad de las organizaciones sociales de desarrollar tareas que involucran la disponibilidad de recursos (tiempo, conocimientos específicos, tecnologías) a los que habitualmente no acceden en tiempo y forma, y que la universidad puede ofrecer de forma eficaz. c) La promoción y el sostenimiento de espacios de aprendizaje dentro de la Universidad donde poner en juego ciertas anticipaciones de las competencias requeridas por las prácticas profesionales de los estudiantes.

Este Laboratorio es una propuesta curricular que se sitúa en un punto intermedio entre la producción de conocimientos - a partir del contacto directo de los estudiantes con su entorno- y la intervención -mediante la realización de un estudio específico planteado por una organización social en función de una problemática que desea abordar y de la comunicación y difusión de sus resultados en las mismas comunidades donde ésta desarrolla sus tareas.

Se parte de la articulación con una organización social y se define junto con ella una necesidad o demanda específica a cuya solución se busca contribuir. Su identificación se construye en los primeros vínculos del equipo docente y los estudiantes con la organización que se trabajará durante el cuatrimestre y se plantea como eje problemático. Sobre esta cuestión se realiza algún tipo de indagación llamada estudio específico: sistematización y/o actualización de información disponible, relevamiento cuantitativo o cualitativo sobre actividades de la organización, estudios de opinión sobre temáticas específicas, entre otras. Los resultados del estudio se "devuelven" a la organización y su comunidad de referencia en formatos comunicacionales pasibles de ser aprovechados por los interesados.

\subsection{Modalidades de trabajo y organización de las tareas}

Esta asignatura se dicta durante un semestre, ocupando 64 horas de trabajo en aula, además de otras 32 de trabajo extraáulico. Está organizada en cuatro etapas o momentos clave; para cada una de ellas se piensan conocimientos necesarios, capacidades específicas a desarrollar y actividades para el trabajo en equipo. En una primera etapa se presentan la problemática general de la Región Metropolitana de Buenos Aires, en especial del Conurbano, y la manera en que la acción de las organizaciones sociales en esta región se relaciona con esta problemática, mostrando además la diversidad de formas organizativas que surgen para intentar dar respuesta a diferentes cuestiones desde distintos sectores sociales. La presentación de los temas enunciados se enfoca desde una perspectiva histórica, económica y política. El enfoque teórico del Laboratorio se sostiene en la hipótesis de que es difícil comprender al mundo asociativo si no se lo relaciona con los modelos de desarrollo hegemónicos, y se propone el análisis de las organizaciones a partir del desarrollo de la relación entre Estado, mercado y sociedad civil en los distintos períodos históricos. Esta es la parte más teórica y expositiva de la materia. En un segundo momento, los estudiantes se aproximan al conocimiento de las organizaciones sociales que participan en la materia (2 o 3 por cuatrimestre), primero a partir de un panel en la que ellas "se presentan" al curso; luego, tomando en cuenta los intereses de los estudiantes, se conforman comisiones de trabajo por organización y se produce colectivamente un informe de caracterización general de esa organización. Esta tarea requiere una o más visitas a la organización en su lugar de trabajo habitual, entrevistas con los referentes, búsqueda y procesamiento de material adicional y una reflexión grupal sobre la organización, retomando los contenidos incorporados en la etapa anterior y planteando sus logros, problemas e innovaciones. En esta etapa se da el mayor acercamiento de los estudiantes a la vida cotidiana de las organizaciones, se comprende lo complejo de su accionar, los condicionamientos que impone el contexto y la creatividad de las 
organizaciones para resolver sus limitaciones. También en este momento se busca que los estudiantes tengan una experiencia intensa de trabajo en equipo, que sirve como antecedente del trabajo más importante "hacia fuera" que sigue en las siguientes etapas. El tercer momento clave es el de la elaboración de un estudio en torno a un eje problemático específico, que se define en conjunto con cada una de las organizaciones sociales involucradas y responde a necesidades de las mismas. Consideramos importante e innovador que sean ellas las que aparecen planteando de algún modo "la agenda de la investigación", invirtiendo sustancialmente la relación tradicional entre universidad (sujeto investigador que recoge los datos y prepara un informe académico que casi nunca retorna a los que brindaron la información) y organización social (objeto de estudio sobre el que se realizan numerosos diagnósticos y pocas veces se ofrecen soluciones a sus problemas concretos). En esta etapa los estudiantes trabajan aplicando elementos de metodología de la investigación social, diseñan encuestas o entrevistas, realizan el trabajo de campo y el procesamiento y análisis de esa información producida. Es el momento de trabajo colectivo más intenso y de apropiación en la práctica de los quehaceres del investigador social.

Por último los estudiantes deben elaborar un producto para comunicar a la organización en cuestión, al resto de sus compañeros y a la comunidad universitaria el trabajo realizado y sus principales conclusiones. Aquí el formato comunicacional de la devolución asume diferentes formas, entre ellas presentaciones de filminas o afiches, videos y fotos, micros de radio, propuestas de folletos para la organización, etc. Para ello se cuenta con especialistas en comunicación que asesoran y acompañan la realización de estos productos.

Finalmente se realiza un encuentro al que se invita a las organizaciones sociales con las que se trabajó, y a otros actores del ámbito académico y del mundo asociativo. En el mismo, se exponen los resultados de los estudios y se abre un espacio de reflexión y evaluación conjunta entre estudiantes, organizaciones y docentes (acerca de lo que fue la experiencia, de las conclusiones y su utilidad para las organizaciones, las posibles estrategias hacia delante, etc.).

\section{Algunas reflexiones sobre la práctica docente en esta experiencia}

La formación que se propone en materias como la que estamos presentando tiene un fuerte componente de aprendizaje en la práctica y junto a otros. Se trata de un tipo específico de formación que introduce elementos de compromiso con el territorio, con las organizaciones sociales y en relación a los integrantes del grupo de estudiantes.

La búsqueda de un punto de encuentro capaz de contemplar las expectativas de las organizaciones sociales y los objetivos de la universidad expresados en la materia es una tarea central y estratégica. De ello depende, en gran medida, la utilidad social de los conocimientos producidos, la formación profesional crítica y propositiva que se pretende y las redes de vínculos que a partir de una intervención específica se generan o potencian.

La utilidad social de los conocimientos producidos, como uno de los elementos estructurantes de la experiencia, deja de ser un principio prescriptivo en tanto se enlaza con un pedido específico de las organizaciones y recibe alternativas de respuesta.

Las preguntas de investigación que se construyen junto con estos actores del escenario metropolitano son los elementos que dan consistencia específica a la propuesta didáctica, a los objetivos, los contenidos conceptuales y metodológicos y los productos finales que enhebran la formación interdisciplinaria que se propone desde el Laboratorio.

Esta experiencia ha construido una propuesta institucional de trabajo interdisciplinar con actores provenientes de diferentes campos, lógicas y capitales. Se trata de un dispositivo que requiere cierto perfil docente para su desarrollo.

¿Qué perfil docente puede orientar experiencias de enseñanza 
por proyectos? ¿Qué vinculación con los estudiantes favorece la "inmersión" en el proceso en este tipo de experiencias? El equipo docente monitorea la producción del trabajo de los estudiantes en el desarrollo del proyecto e interviene cuando lo considera necesario para fortalecer el trabajo que el grupo está realizando. Simultáneamente, se proponen actividades que favorecen la autoevaluación de los grupos y se realiza un seguimiento de cada integrante.

En efecto, un esfuerzo importante de los docentes se orienta a ponerse en un lugar de acompañamiento de la tarea de los estudiantes con las organizaciones, sin dirigir o imponerse unívocamente, ni sumarse al grupo de estudiantes como uno más. Se generan condiciones para que el grupo tome decisiones tanto en términos de la planificacion de las tareas como en el contenido y el formato de los productos acordados con la organización social. Los estudiantes son interpelados para participar tanto en las sesiones de plenario como en los encuentros de trabajo de cada comisión y en los intercambios con las organizaciones sociales. Este rol docente, sus alcances, el tipo de acompañamiento generado, los matices en las decisiones frente a las tareas, el modo de "leer" las características del grupo, han ido delineándose a medida que el Laboratorio avanzó como experiencia. Se configuró inicialmente en forma de aproximación intuitiva, en oposición a un modo tradicional del quehacer docente en el ámbito universitario.

El del Laboratorio es un equipo docente con experiencia de trabajo con estudiantes del primer tramo universitario, así como con organizaciones sociales, aunque sin formación didáctica específica para este tipo de materias; dicha formación se fue construyendo con el tiempo. Este proceso supuso la puesta a prueba y elección de distintas modalidades de trabajo con los estudiantes y las organizaciones sociales. Asimismo, estimuló la introducción de mejoras, ajustes y traducciones de la propuesta, en función de los desafíos y la dinámica que se generan en los diferentes momentos de la cursada.

La priorización del trabajo colectivo del equipo docente atraviesa transversalmente la experiencia sistematizada. Se pone en juego tanto al pensar la programación como al analizar las alternativas para resolver problemas derivados de los cursos de acción planificados y al asumir la incorporación de docentes.

El equipo docente considera el trabajo en equipo de los estudiantes como un desafío en el aprendizaje, en términos de las competencias profesionales que el Laboratorio propone. En las reflexiones colectivas del equipo docente sobre los avances de cada grupo y en el análisis de situaciones conflictivas específicas ha mantenido la preocupación por instalar la necesidad del trabajo en grupo, ha explorado distintas estrategias para conseguirlo, ha comparado situaciones diferentes hasta distinguir condiciones contextuales que favorecieran esta dinámica. Entre las condiciones que contribuyen a dinamizar la construcción del grupo, se destaca la generación de espacios de autoevaluación, así como los distintos momentos de reflexión colectiva con acompañamiento del docente al cierre de cada una de las etapas en que está organizado el trabajo, tendientes a reorientar el curso del proceso y a reasignar tareas y roles entre los estudiantes.

Otro aspecto destacado es el aprendizaje de alternativas para el manejo de la incertidumbre y la imprevisión como situaciones en las que los docentes se respaldan y fortalecen. Al desarrollar una tarea que supone una negociación permanente entre varios actores, donde el desenlace positivo no está garantizado, se consolidó desde el inicio una actitud de apertura para los cambios y para la búsqueda creativa de alternativas que ha estimulado el deseo de ampliar la formación como docentes, así como el de dialogar con otros acerca de los desafíos del Laboratorio.

En el Laboratorio, la reflexión permanente sobre las estrategias seleccionadas y las reorientaciones de los cursos de acción se resuelven en un espacio común de consulta y discusión, altamente valorado por el equipo docente. Al mismo tiempo, la comunicación a los estudiantes de las intenciones expresadas en la programación curricular constituye una instancia importante al iniciar el dictado de la materia.

En este sentido, el programa, con su carácter "abierto", opera como un marco sustantivo de referencia que permite ganar flexibilidad en el proceso, ya que la dinámica del Laboratorio exige a los docentes la revisión y actualización de los contenidos y la articulación de recursos y actores en escenarios cambiantes, tanto en función de la planificación curricular como de la naturaleza del objeto de estudio e intervención. A esto se suma la realización de acuerdo de trabajos claros y consistentes con cada una de las organizaciones con las que se va a trabajar cada semestre.

La experiencia del Laboratorio se desarrolla en un escenario de turbulencias e incertidumbres propio del trabajo de las organizaciones sociales. Los cambios de rumbo, una crisis institucional, modificaciones particulares del entorno pueden tener implicancias directas en el desarrollo de la cursada y en el cronograma de trabajo. Por eso el programa opera siempre como una hipótesis de trabajo que expresa las condiciones en las que se desarrollará la tarea, ofreciendo una especie de cartografía a la que es posible recurrir para buscar información o para reorientar el proceso. Se trata de un recurso utilizado cotidianamente por el equipo docente para organizar la tarea cuya flexibilidad se complementa con los tránsitos alternativos por la materia, que habilita incorporar la incertidumbre como parte del proceso.

En clave de la propuesta didáctica, se problematiza el modo en que aprenden los estudiantes, y los contenidos que trabajan. Por un lado, se posibilita la problematización, integración y contrastación de los saberes construidos en los distintos campos de formación. Asimismo, los estudiantes participan y protagonizan la decisión de qué y cómo hacer. Esto les permite tener una noción diferente, 


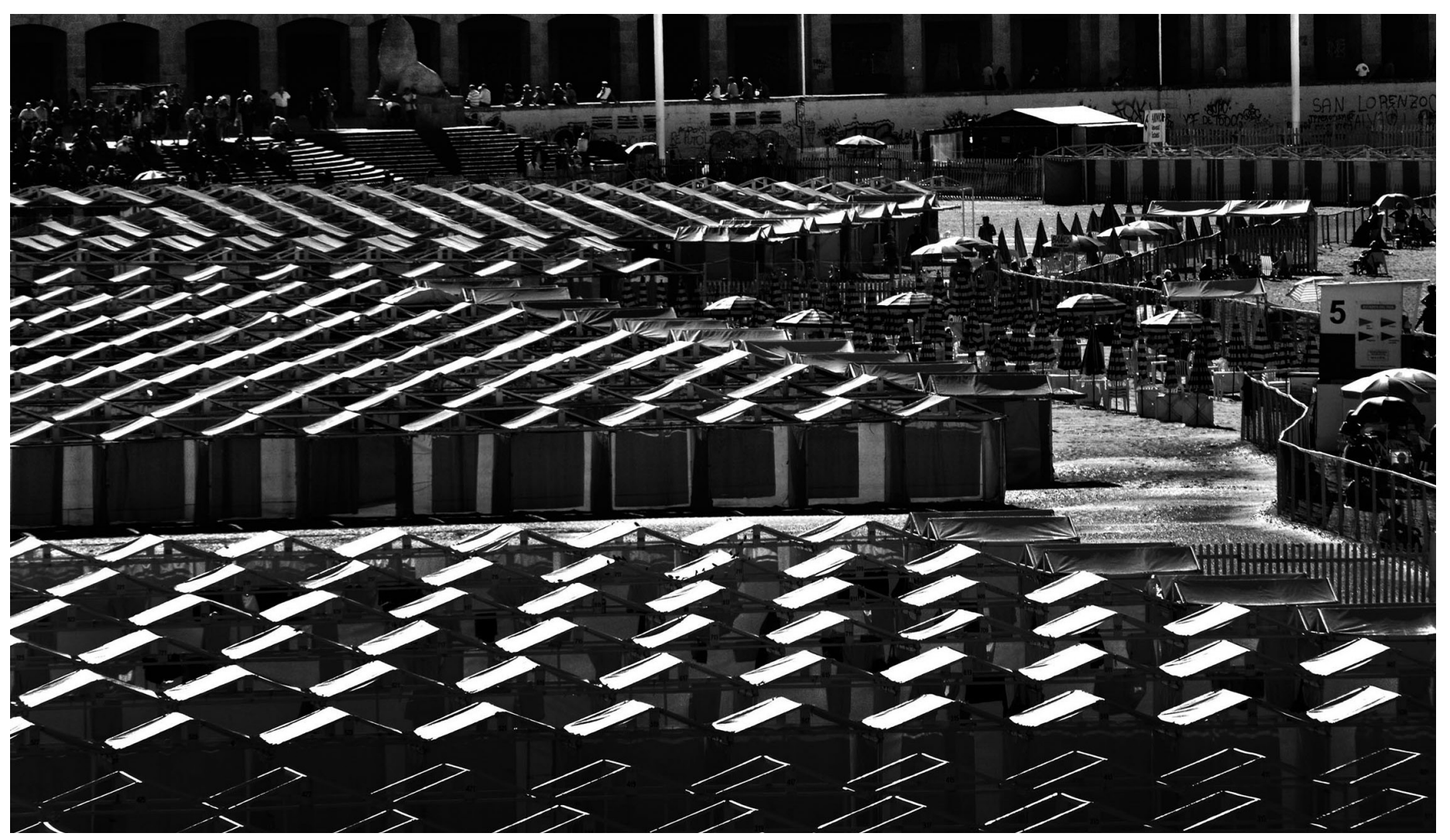

(c) Oscar Dechiara

más completa, de la relación producto-proceso, ya que es una experiencia que muestra complejidades, tensiones, conflictos, potencialidades y una planificación que se concreta en la práctica. Destacamos, por último, los aprendizajes de los estudiantes en su paso por esta materia. Pueden ordenarse en distintos planos: en torno a la gestión de proyectos; en torno a la interacción con otros (compañeros, docentes, miembros de las organizaciones, vecinos de los barrios) dentro y fuera de la universidad, lo que supone generación de acuerdos, situaciones de negociación, manejo de situaciones conflictivas y liderazgo; en relación con el uso de distintos espacios que ofrece la universidad (biblioteca, laboratorios de Sistemas de Información Geográfica y de Comunicación, salas de informática, áreas de investigación de los institutos); en torno de la evaluación de proceso y autoevaluación. Otros elementos destacados por los propios estudiantes son el compromiso social y la movilización política que genera esta propuesta innovadora de trabajo fuera del aula y para otros, el cambio en su actitud en otras materias, frente a otros contenidos y otros docentes. Destacan esto en el marco del valor que le dan al compromiso que asume la universidad al trabajar con otros actores del territorio y el hecho de que esa experiencia los involucre a ellos mismos en representación de la institución.
Los estudiantes experimentan una de las formas posibles del desempeño profesional: aquel que puede hacer aportes específicos en escenarios amplios, conflictivos y complejos; abriendo la posibilidad de aliarse con actores sociales, políticos y económicos que protagonizan y dinamizan los procesos de transformación social y de ampliación democrática.

Las "marcas" que imprime el Laboratorio tanto en las organizaciones como en los estudiantes son señales potentes para repensar la relación entre la universidad y los procesos de transformación colectiva. En términos de la educación universitaria, el principal logro es la formación de futuros profesionales que puedan pensarse realizando aportes específicos en proyectos sociales, políticos y económicos capaces de poner en el centro de atención el desarrollo de su territorio y la construcción colectiva

\section{Referencias bibliográficas}

Abramovich, A.; Da Representaçao, N.; Fournier, M.; (Coords.) (2012). Aprender haciendo con otros. Una experiencia de formación universitaria con organizaciones sociales. Buenos Aires: UNGS. 